\title{
Jeans' gravitational instability and nonextensive kinetic theory
}

\author{
J. A. S. Lima, R. Silva, and J. Santos
}

Departamento de Física, UFRN, C.P. 1641, 59072-970 Natal, RN, Brazil

Received 18 March 2002 /Accepted 27 August 2002

\begin{abstract}
The concept of Jeans gravitational instability is rediscussed in the framework of nonextensive statistics and its associated kinetic theory. A simple analytical formula generalizing the Jeans criterion is derived by assuming that the unperturbed self-gravitating collisionless gas is kinetically described by the $q$-parameterized class of power law velocity distributions. It is found that the critical values of wavelength and mass depend explicitly on the nonextensive $q$-parameter. The standard Jeans wavelength derived for a Maxwellian distribution is recovered in the limiting case $q=1$. For power-law distributions with cutoff, the instability condition is weakened with the system becoming unstable even for wavelengths of the disturbance smaller than the standard Jeans length $\lambda_{\mathrm{J}}$.
\end{abstract}

Key words. gravitation - hydrodynamics

\section{Introduction}

In a seminal paper, James Jeans discussed the conditions under which a fluid becomes gravitationally unstable under the action of its own gravity (Jeans 1929). Nowadays, for any relevant length scales in the Universe (stars, galaxies, clusters, etc.), such an instability has been recognized as the key mechanism to explain the gravitational formation of structures and their evolution in the linear regime.

A noteworthy conclusion of Jeans' work is that perturbations with mass greater than a critical value $M_{\mathrm{J}}$ (Jeans' mass) may grow thereby producing gravitationally bounded structures, whereas perturbations with a mass smaller than $M_{\mathrm{J}}$ do not grow and behave like acoustic waves. This gravitational instability criterion remains basically valid and plays a fundamental role even in the expanding Universe (Peebles 1993; Coles \& Lucchin 1995). In terms of the wavelengths of a fluctuation, Jeans' criterion says that $\lambda$ should be greater than a critical value $\lambda_{\mathrm{J}}=\sqrt{\pi v_{\mathrm{S}}^{2} /\left(G \rho_{0}\right)}$, which is named the Jeans length. In this formula $G$ is the gravitational constant, $\rho_{0}$ is the unperturbed matter density and $v_{\mathrm{s}}$ is the sound speed for adiabatic perturbations. As is widely known, the same criterion also holds for a collisionless self-gravitating cloud of gas, except that the sound speed $v_{\mathrm{s}}$ is replaced by the velocity dispersion $\sigma$. As explained in many textbooks (see, for instance, Binney \& Tremaine 1987), such a result follows naturally from a kinetic theoretical approach based on the the Vlasov equation, where the evolution of the collisionless gas is ultimately described by perturbing the equilibrium Maxwellian velocity distribution.

Send offprint requests to: J. A. S. Lima, e-mail: limajas@dfte.ufrn.br
On the other hand, some recent studies involving the statistical description of a large variety of physical systems revealed that some extension of the standard Boltzmann-Gibbs approach should be needed. Particular examples are systems endowed with long duration memory, anomalous diffusion, turbulence in pure-electron plasma, self-gravitating systems or more generally systems endowed with long range interactions. In order to work with such problems, Tsallis (1988) proposed the following generalization of the Boltzmann-Gibbs (BG) entropy formula for statistical equilibrium (see also http.tsallis.cat.cbpf.br/biblio.htm for an extensive and updated list of problems and references)

$S_{q}=-k_{\mathrm{B}}\left(1-\sum_{i} p_{i}^{q}\right) /(q-1)$,

where $k_{\mathrm{B}}$ is the Boltzmann constant, $p_{i}$ is the probability of the $i$ th microstate and $q$ is a parameter quantifying the degree of nonextensivity. In the limit $q \rightarrow 1$ the celebrated BG extensive formula, namely

$S=-k_{\mathrm{B}} \sum_{i} p_{i} \ln p_{i}$,

is recovered. One of the main properties of $S_{q}$ is its pseudoadditivity. Given a composite system $A+B$, constituted by two subsystems $A$ and $B$, which are independent in the sense of factorizability of the microstate probabilities, the Tsallis measure verifies $S_{q}(A+B)=S_{q}(A)+S_{q}(B)+(1-q) k_{\mathrm{B}}^{-1} S_{q}(A) S_{q}(B)$. In the limit $q \rightarrow 1, S_{q}$ reduces to the standard logarithmic measure, and the usual additivity of the extensive BG statistical mechanics and thermodynamics is also recovered. Thus, if $q$ differs from unity, the entropy becomes nonextensive (superextensive if $q<1$, and subextensive if $q>1$ ), with the Boltzmann factor 
generalized into a power law. In other words, $|q-1|$ quantifies the lack of extensivity of the system.

A large portion of the experimental evidence supporting Tsallis proposal involves a non-Maxwellian (power-law) equilibrium distribution function associated with the thermostatistical description of the classical $N$-body problem. This equilibrium velocity $q$-distribution may be derived from at least three different methods: (i) through a simple nonextensive generalization of the Maxwell ansatz, which is based on the isotropy of the velocity space (Silva et al. 1998); (ii) within the nonextensive canonical ensemble, that is, maximizing Tsallis entropy under the constraints imposed by normalization and the energy mean value (Abe 1999) (iii) using a more rigorous treatment based on the nonextensive formulation of the Boltzmann $H$-theorem (Lima et al. 2001).

In the astrophysical context, the nonextensive equilibrium velocity distribution related to Tsallis' statistics has been applied to stellar collisionless systems (Plastino \& Plastino 1993), as well as to the peculiar velocity function of galaxies clusters (Lavagno 1998). In the former paper, the entropy nonextensive formula was maximized taking into account the constraints imposed by the total mass and energy density. It was found that the solutions behave like stellar polytropes and that the mass is finite if the nonextensive parameter is bounded by $q \leq 9 / 7$. More recently, a more detailed account for spherically symmetric systems involving either the gravothermal instability (Taruya \& Sakagami 2002; Chavanis 2002), or the complete determination of the density profiles has been presented in the literature (Lima \& de Souza 2002).

Applications in plasma physics are also important in their own right, both from a methodological and physical viewpoint. In this case, some metastable states in pure electron plasmas, and the dispersion relations for an electrostatic plane-wave propagation in a collisionless thermal plasma (including undamped Bohm-Gross and Landau damped waves) have also been studied and compared to the standard results (Boghosian 1996; Lima et al. 2000). In particular, Liu et al. (1994) showed a reasonable indication for the non-Maxwellian velocity distribution from plasma experiments. All this empirical evidence deal directly or indirectly, with the $q$-distribution of velocities for a nonrelativistic gas.

In the present work, we quantify to what extent the nonextensive effects modify the gravitational instability criterion established by Jeans. For this enlarged framework, we deduce a new analytical expression for the Jeans dispersion relation which gives rise to an extended instability criterion in accordance with Tsallis thermostatistics.

\section{Nonextensive gravitational instability}

In what follows, we focus our attention on the kinetic description of a many particle system within the nonrelativistic gravitational context. More precisely, we consider an infinite self-gravitating collisionless gas described by a distribution function $f(\boldsymbol{v}, \boldsymbol{r}, t)$ slightly depart from equilibrium. If $f_{0}(v)$ corresponds to the unperturbed homogeneous and timeindependent equilibrium distribution, sometimes referred to as the "Jeans Swindle", the resulting particle distribution function may be approximated as

$f=f_{0}(v)+f_{1}(\boldsymbol{v}, \boldsymbol{r}, t), \quad f_{1} \ll f_{0}$,

where $f_{1}$ is the corresponding perturbation in the distribution function. Following standard lines, the dynamic behavior of this system can be described by the linearized Vlasov and Poisson equations. By neglecting up to second-order terms in the expansion of the distribution function one obtains

$\frac{\partial f_{1}}{\partial t}+\boldsymbol{v} \cdot \frac{\partial f_{1}}{\partial \boldsymbol{r}}=\nabla \phi_{0} \cdot \frac{\partial f_{1}}{\partial \boldsymbol{v}}+\nabla \phi_{1} \cdot \frac{\partial f_{0}}{\partial \boldsymbol{v}}$

$\nabla^{2} \phi_{1}=4 \pi G \int f_{1}(\boldsymbol{r}, \boldsymbol{v}, t) \mathrm{d}^{3} v$

where $\phi_{0}(\boldsymbol{r})$ and $\phi_{1}(\boldsymbol{r})$ are, respectively, the unperturbed gravitational potential and its first order correction. As is well known, if the "Jeans Swindle" is assumed, one may set the unperturbed potential $\phi_{0}=0$. In this case, the solutions of the above equations can be written as $f_{1}(\boldsymbol{r}, \boldsymbol{v}, t)=F(\boldsymbol{v}) \exp \{i(\boldsymbol{k} \cdot \boldsymbol{r}-\omega t)\}$ and $\phi_{1}(\boldsymbol{r}, t)=\phi \exp \{i(\boldsymbol{k} \cdot \boldsymbol{r}-$ $\omega t)$ \} provided that $F(v)$ and $\phi$ satisfy the relations

$(\boldsymbol{k} \cdot \boldsymbol{v}-\omega) F(\boldsymbol{v})-\phi \boldsymbol{k} \cdot \frac{\partial f_{0}}{\partial \boldsymbol{v}}=0$,

$k^{2} \phi=-4 \pi G \int F(\boldsymbol{v}) \mathrm{d}^{3} v$.

Combining these expressions one obtains the dispersion relation between $\omega$ and $\boldsymbol{k}$

$1+\frac{4 \pi G}{k^{2}} \int \frac{\boldsymbol{k} \cdot \partial f_{0} / \partial \boldsymbol{v}}{\boldsymbol{k} \cdot \boldsymbol{v}-\omega} \mathrm{d}^{3} v=0$

The standard instability analysis follows from the above dispersion relation when one takes $f_{0}(\boldsymbol{v})$ as the Maxwellian velocity distribution.

Consider now the $q$-nonextensive framework proposed by Tsallis. In this case, the equilibrium distribution function $f_{0}(\boldsymbol{v})$ can be written as (Silva et al. 1998; Lima et al. 2000)

$f_{0}(\boldsymbol{v})=\frac{\rho_{0} B_{q}}{\left(2 \pi \sigma^{2}\right)^{3 / 2}}\left[1-(q-1) \frac{v^{2}}{2 \sigma^{2}}\right]^{\frac{1}{q-1}}$,

where the normalization constant reads

$B_{q}=\frac{(3 q-1)(q+1)}{4} \frac{\sqrt{q-1} \Gamma\left(\frac{1}{q-1}+\frac{1}{2}\right)}{\Gamma\left(\frac{1}{q-1}\right)}$

for $q \geq 1$, and

$B_{q}=\left(\frac{3 q-1}{2}\right) \frac{\sqrt{1-q} \Gamma\left(\frac{1}{1-q}\right)}{\Gamma\left(\frac{1}{1-q}-\frac{1}{2}\right)}$

for $\frac{1}{3}<q<1$. Here $\sigma$ is the velocity dispersion and $\rho_{0}$ is the equilibrium density. As one may check, for $q<1 / 3$, the $q$ distribution (9) is unnormalizable, while for $q>1$, it exhibits a 
thermal cutoff on the maximum value allowed for the velocity of the particles, which is given by

$v_{\max }=\sqrt{\frac{2 \sigma^{2}}{q-1}}$.

This thermal cut-off is absent when $q \leq 1$, that is, $v_{\max }$ is also unbounded for these values of the $q$-parameter. In this connection, it is worth noticing that the spirit of the $H$-theorem is totally preserved for this nonextensive velocity distribution. As a matter of fact, by introducing a generalized collisional term, $C_{q}(f)$, it has been shown that the entropy source is definite positive for $q>0$, and does not vanish unless the $q$ equilibrium distribution function assumes the above power-law form (Lima et al. 2001). In addition, taking into account that $\lim _{|z| \rightarrow \infty} \Gamma(z+a) /\left[z^{a} \Gamma(z)\right]=1$, as well as that $\lim _{q \rightarrow 1}[1-(q-$ 1) $\left.x^{2}\right]^{1 /(q-1)}=\exp \left(-x^{2}\right)$ (Abramowitz \& Stegun 1972), the expressions (10) and (11) defining $B_{q}$ reduce to $B_{1}=1$, and as should be expected the distribution function $f_{0}$ reduces to the exponential Maxwellian distribution

$f_{0}(\boldsymbol{v})=\frac{\rho_{0}}{\left(2 \pi \sigma^{2}\right)^{3 / 2}} \exp \left(-\frac{v^{2}}{2 \sigma^{2}}\right)$.

In Fig. 1 we have plotted the nonextensive distribution as a function of $v$ for some selected values of the $q$ parameter. As can be seen, for $q>1$ the distribution exhibits a cutoff on the maximum value allowed for the velocity of the particles. In the limit $q \rightarrow 1$ we see from (12) that $v_{\max } \rightarrow \infty$ and the power-law behavior reduces to the exponential Maxwellian distribution. For $q<1$ the cutoff is also absent with the curves decreasing less rapidly than in the Maxwellian case. Note also the convergence from power-law to the standard Gaussian curve as long as the $q$ parameter approaches unity.

Now, substituting the nonextensive equilibrium distribution given by (9) into the dispersion relation (8) one finds

$1-\frac{4 \pi G \rho_{0}}{\sigma^{5}} \frac{B_{q}}{(2 \pi)^{3 / 2}} \int \frac{v_{x}\left[1-(q-1) \frac{v^{2}}{2 \sigma^{2}}\right]^{\frac{2-q}{q-1}}}{k v_{x}-\omega} \mathrm{d}^{3} v=0$

where the $v_{x}$ axis has been chosen in the direction of $\boldsymbol{k}$.

In the study of Jeans instability, the boundary between stable and unstable solutions is achieved by setting $\omega=0$ in (14). For this value of $\omega$ the above integral can be easily evaluated and the result is a $q$-parameterized family of critical wavenumbers $k_{q}$ given by

$k_{q}^{2}=k_{\mathrm{J}}^{2} \frac{(3 q-1)}{2}$

where $k_{\mathrm{J}}^{2}=4 \pi G \rho_{0} / \sigma^{2}$ is the classical Jeans wavenumber (Binney \& Tremaine 1987). From (15) we have the $q$ wavelength $\lambda_{q}=2 \pi / k_{q}=\lambda_{\mathrm{J}} \sqrt{2 /(3 q-1)}$ where $\lambda_{\mathrm{J}}$ is the Jeans wavelength. Note that the classical value of the Jeans wavenumber and wavelength as obtained from fluid theory are recovered only if $q=1$. For $q>1(1 / 3<q<1)$ the $q$-wavelength $\lambda_{q}$ is decreased (increased) by the factor $\sqrt{2 /(3 q-1)}$. Associated with $\lambda_{q}$ there is a critical mass $M_{q}$, defined as the mass contained within a sphere of diameter $\lambda_{q}$, which is given by $M_{q}=\frac{4 \pi}{3} \rho_{0}\left(\lambda_{q} / 2\right)^{3}=M_{\mathrm{J}}[2 /(3 q-1)]^{3 / 2}$,

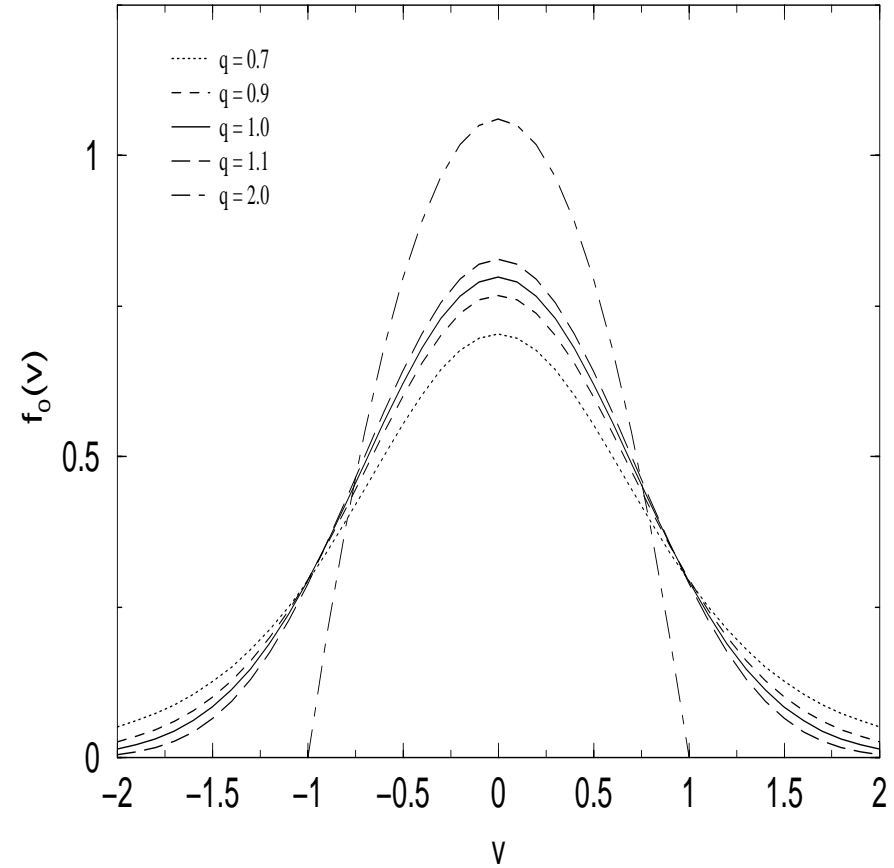

Fig. 1. Nonextensive velocity distribution function $f_{0}(\boldsymbol{v})$ for typical values of the $q$ parameter. Power law distributions with $q>1$ exhibits a thermal cutoff on the maximum value allowed for the velocity of the particles. This cutoff is absent for $\frac{1}{3}<q \leq 1$, and the distribution is unnormalizable for $q<\frac{1}{3}$.

where $M_{\mathrm{J}}$ is the critical Jeans mass. Similarly, we see that for $q>1(1 / 3<q<1)$ the critical mass is decreased (increased) by the factor $[2 /(3 q-1)]^{3 / 2}$. Therefore, in comparison with a Maxwellian gas $(q=1)$, a nonextensive collisionless selfgravitating system is more (or less) unstable depending on the value assumed by the $q$-parameter.

Let us now discuss in more detail the unstable modes. As happens in the perturbed fluid theory, fluctuations with wavelengths $\lambda>\lambda_{\mathrm{J}}$ will be unstable. In order to check what happens in the present kinetic approach we set $\omega=i \gamma$, where $\gamma$ is real positive, and insert this into the dispersion relation (14). As before, choosing the $v_{x}$ axis in the direction of $\boldsymbol{k}$, the following dispersion relation is obtained

$\frac{k^{2}}{k_{q}^{2}}=1-\sqrt{\pi} \beta^{2} I_{q}(\beta)$,

where $\beta=\gamma / \sqrt{2} k \sigma$ and $I_{q}(\beta)$ is a $q$-dependent integral given by

$$
\begin{aligned}
I_{q}(\beta)= & \frac{\sqrt{q-1} \Gamma\left(\frac{1}{q-1}+\frac{1}{2}\right)}{\Gamma\left(\frac{1}{q-1}\right)} \frac{q+1}{\pi} \\
& \times \int_{0}^{\frac{1}{\sqrt{q-1}}} \frac{\left[1-(q-1) x^{2}\right]^{\frac{1}{q-1}}}{\beta^{2}+x^{2}} \mathrm{~d} x
\end{aligned}
$$

for $q>1$ and

$I_{q}(\beta)=\frac{\sqrt{1-q} \Gamma\left(\frac{1}{1-q}\right)}{\Gamma\left(\frac{1}{1-q}-\frac{1}{2}\right)} \frac{2}{\pi} \int_{0}^{\infty} \frac{\left[1-(q-1) x^{2}\right]^{\frac{1}{q-1}}}{\beta^{2}+x^{2}} \mathrm{~d} x$ 


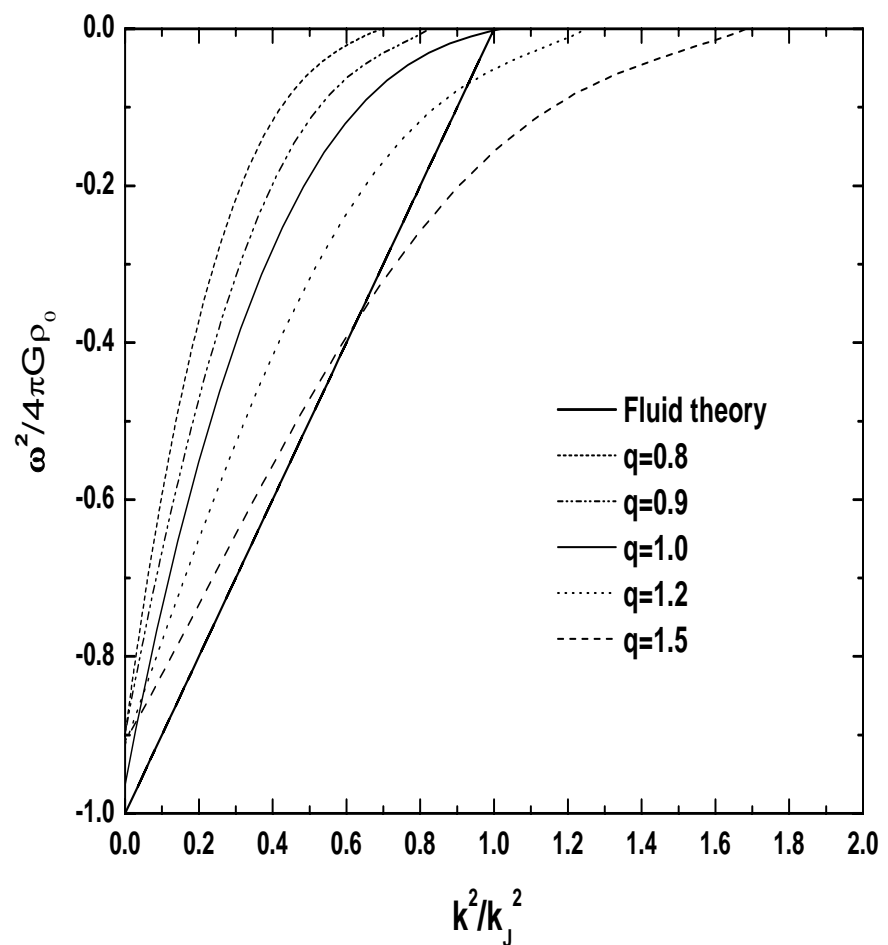

Fig. 2. Unstable branches of the dispersion relations for an infinite self-gravitating collisionless gas obeying the nonextensive Tsallis $q$ statistics. For comparison the straight line representing the dispersion relation for an infinite homogeneous fluid has also been plotted. We see that for $q>1$ (power-law distributions with cutoff), the system is unstable even for wavelengths smaller than the standard Jeans length value $\left(\lambda_{\mathrm{J}}\right)$

for $1 / 3<q<1$. It should be noticed that the limit $q \rightarrow 1$ of both expressions is

$\lim _{q \rightarrow 1} I_{q}(\beta)=\frac{2}{\pi} \int_{0}^{\infty} \frac{\mathrm{e}^{-x^{2}}}{\beta^{2}+x^{2}} \mathrm{~d} x=\frac{\mathrm{e}^{\beta^{2}}}{\beta}[1-\operatorname{erf}(\beta)]$,

with the expression (16) reducing to

$\frac{k^{2}}{k_{\mathrm{J}}^{2}}=1-\sqrt{\pi} \beta \mathrm{e}^{\beta^{2}}[1-\operatorname{erf}(\beta)]$,

which is the standard dispersion relation for stellar systems (Binney \& Tremaine 1987). For an arbitrary value of $q$, the dispersion relation is given explicitly by the integral form (16) which can be numerically evaluated. In Fig 2 we plot the unstable branches for several values of $q$ along with the dispersion relation for an infinite homogeneous fluid which is given by the solid straight line (see Binney \& Tremaine 1987 for comparison).

As can be seen from these plots, for $q>1$ the system presents instability even for wavenumbers of the disturbance greater than the standard critical Jeans value $\left(k_{\mathrm{J}}\right)$. In other words, this kind of $q$-gaseous system is more unstable than the one described by the standard Maxwellian curve, and therefore, structures are more easily formed in this nonextensive context. However, for $q<1$ the system may remain stable even for disturbance with a wavenumber smaller than the Jeans wavenumber. This yields a generalization of the Jeans gravitational

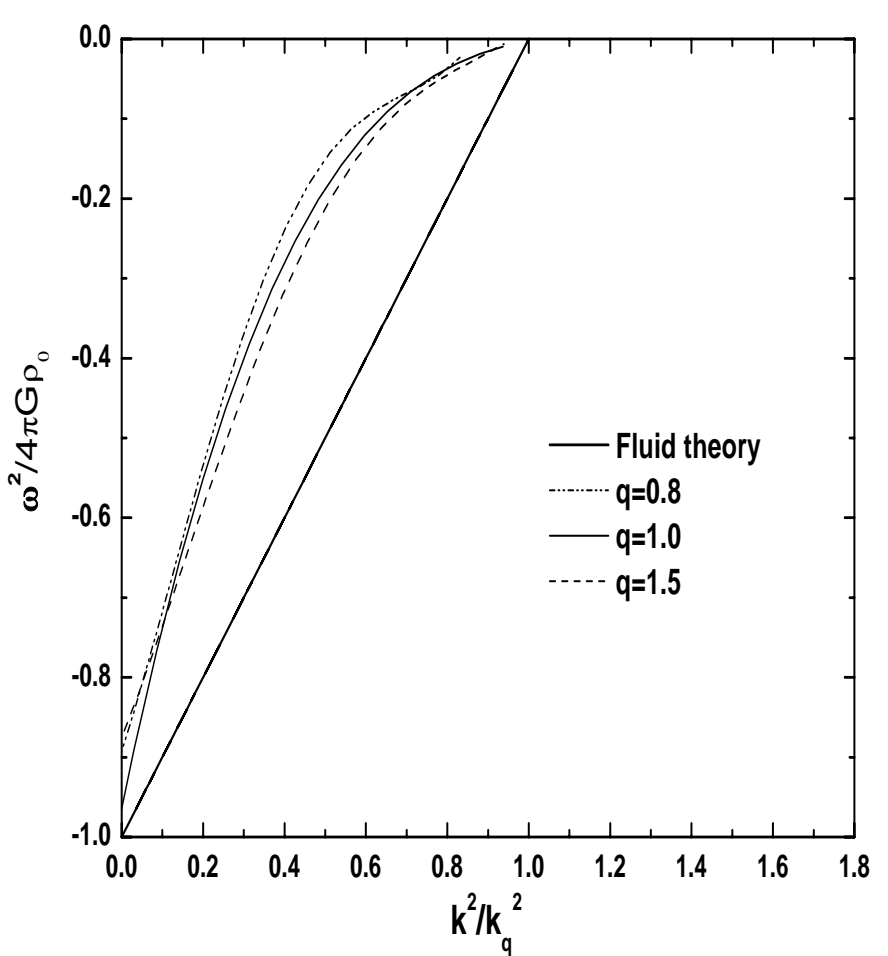

Fig. 3. Unstable branches of the dispersion relations as a function of the critical wavenumbers $k_{q}$. Notice that the magnitude of $k_{q}$ is related to the critical Jeans wavenumber by Eq. (15). As in Fig. 2, the straight solid line stands to the case of an infinite homogeneous fluid.

instability mechanism within Tsallis' nonextensive thermostatistical context. The standard Jeans criterion is thus modified with critical values of mass and length depending explicitly on the nonextensive $q$-parameter.

In Fig. 3 new plots of the dispersion relation (unstable branches) are presented. Different from Fig. 2, instead of the ratio involving the critical Jeans wavenumber $\left(k_{\mathrm{J}}\right)$, the behavior of the unstable branches are analyzed as a function of the ratio $k / k_{q}$.

As should be expected at first sight, the curves are convergent for the critical ratio $\left(k / k_{q}=1\right)$, but the growth rates are slightly different for each value of $q$. Note also that for all values of $q$, the results are quite different to what happens in the macroscopic fluid approach for gravitational instability (solid straight line).

\section{Final remarks}

We have discussed the Jeans gravitational instability along the lines of the nonextensive statistical formalism proposed by Tsallis. In this extended kinetic framework, the Gaussian phase space density is replaced by a family of power law distributions parameterized by the nonextensive $q$-parameter. An attractive feature of Tsallis thermostatistics is that the models are analytically tractable in such a way that a detailed comparison with the extensive Maxwell-Boltzmann approach is readily achieved.

The main interest of our results rests on the fact that the Maxwellian curve may provide only a very crude description of the velocity distribution for a self-gravitating gas, or more 
generally for any system endowed with long range interactions. As we have seen, a well determined criterion for gravitational instability is not a privilege of the exponential velocity distribution function, but is shared by an entire family of power-law functions (named $q$-exponentials) which includes the standard Jeans result for the Maxwellian distribution as a limiting case $(q=1)$. In general, the basic instability criterion is maintained: perturbations with $k>k_{q}$ do not grow (or are damped even considering that the collisionless $q$-gas is a time reversible system), while instability takes place for $k<k_{q}$. However, unlike Jeans' treatment, in this context there exists a family of growth rates parameterized by the nonextensive $q$-parameter. In particular, for $q>1$ (power-law distributions with cutoff) the system presents instability even for wavenumbers of the disturbance greater than the standard critical Jeans value $k_{\mathrm{J}}$.

Finally, since the gravitational instability Jeans' criterion remains basically true for an expanding Universe (Coles \& Lucchin 1995), the adoption of a power law distribution and the resulting nonextensive effects may have interesting consequences for the galaxy formation process. Such implications will be examined in a forthcoming communication.

Acknowledgements. The authors are grateful to Dr. I. Szapudi for many valuable comments. This work was partially supported by the project Pronex/FINEP (No. 41.96.0908.00), FAPESP (00/06695-0), and CNPq (62.0053/01-1-PADCT III/Milenio).

\section{References}

Abramowitz, M., \& Stegun, I. A. 1972, Handbook of Mathematical Functions (Dover Publications)

Abe, S. 1999, Phys. A, 269, 403

Boghosian, B. M. 1996, Phys. Rev. E, 53, 4754

Binney, J., \& Tremaine, S. 1987, Galactic Dynamics (Princeton U. Press, Princeton)

Chavanis, P. H. 2002 [astro-ph/0207080]

Coles, P., \& Lucchin, F. 1995, Cosmology - The Origin and Evolution of Cosmic Structure (John Wiley \& Sons)

Jeans, J. H. 1929, Astronomy and Cosmogony (University Press, Cambridge)

Lavagno, A., Kaniadakis, G., Rego-Monteiro, M., Quarati, P., \& Tsallis, C. 1998, Astroph. Lett. and Comm., 35, 449

Lima, J. A. S., \& de Souza, R. 2002, submitted for publication

Lima, J. A. S., Silva, R., \& Plastino, A. R. 2001, Phys. Rev. Lett., 86, 2938

Lima, J. A. S., Silva, R., \& Santos, J. 2000, Phys. Rev. E, 61, 3260

Liu, J. M., De Groot, J. S., Matte, J. P., Johnston, T. W., \& Drake, R. P. 1994, Phys. Rev. Lett., 72, 2717

Plastino, A., \& Plastino, A. R. 1993, Phys. Lett. A, 177, 177

Peebles, P. J. E. 1993, Principles of Physical Cosmology (Princeton U. Press, Princeton)

Silva, R., Plastino, A. R., \& Lima, J. A. S. 1998, Phys. Lett. A, 249, 401

Taruya, A., \& Sakagami, M. 2002, Physica A, 307, 185

Tsallis, C. 1988, J. Stat. Phys., 52, 479 